04

\title{
Комментарий к статье А.И. Григорьева и С.О. Ширяевой „Оценка интенсивности электромагнитного излучения шаровой молнии“ (ЖТФ. 2019. Т. 89. Вып. 1. С. 22-26.)
}

\section{(C) М.Л. Шматов}

Физико-технический институт им. А.Ф.Иоффре РАН, 194021 Санкт-Петербург, Россия

e-mail: M.Shmatov@mail.ioffe.ru

Поступило в Редакцию 29 октября 2019 г.

В окончательной редакции 6 ноября 2019 г.

Принято к публикации 15 февраля 2020 г.

DOI: $10.21883 /$ JTF.2020.08.49553.356-19

В работе [1] сделана попытка оценить в рамках некоторой модели несколько параметров шаровой молнии на основании сообщений о том, что пролет шаровой молнии рядом с выключенной лампой накаливания вызывал ее свечение, прекращающееся при последующем удалении шаровой молнии. При этом предполагается, что обсуждаемый эффект обусловлен нагревом нити накаливания токами, индуцированными электромагнитной волной, испускаемой шаровой молнией [1]. Ниже показано, что работа [1] содержит как минимум две принципиально важных ошибки, обесценивающих значительную часть представленных в ней результатов.

Интенсивность I электромагнитного излучения, испускаемого зарядом величиной $q$ при движении с ускорением $a$, рассчитана в работе [1] по формуле

$$
I=\frac{q^{2} a^{2}}{6 \pi \varepsilon_{0} c^{3}},
$$

где $\varepsilon_{0}$ - электрическая постоянная, $c-$ скорость света в вакууме. Это соответствует использованию термина „интенсивность“ в смысле ,энергия, излучаемая за единицу времени“ [2,3], что видно, прежде всего, из размерности. В работе [1], где формула (1) введена под номером пять и для описания шаровой молнии используется сокращение ШМ, сделано следующее утверждение: „Чтобы интенсивность излучения, рассчитываемую по формуле (5), можно было сравнить с интенсивностью излучения у поверхности ШМ $I \sim 0.1 \mathrm{~W}$, последнюю нужно умножить на коэффициент $X$, учитывающий ослабление напряженности поля в волне с расстоянием. Численное значение этого коэффициента определится выражением $X \sim\left(L_{2} / L_{1}\right)^{2}$, если принять, что (5) дает интенсивность поля в волне на расстоянии $L_{1}$, а расстояние от ШМ до лампочки $L_{2}$. Принимая $L_{1} \sim 1000 \mathrm{~m}$, $L_{2} \sim 0.1 \mathrm{~m}$, получим $X \sim 10^{-8}$ “. Подход, сформулированный в данном утверждении, противоречит физическому смыслу формулы (1), а его использование обесценивает все приведенные в работе [1] оценки характерных значений $a$ и основанные на них качественные выводы.
Подчеркнем, что представленная в приведенной выше цитате величина $I \sim 0.1 \mathrm{~W}$, где знак „ “ “ имеет, вероятно, значение „порядка“ (или, говоря более подробно, „является величиной порядка“), получена в работе [1] по формуле, соответствующей использованию термина „интенсивность“ в смысле „энергия излучения, проходящего за единицу времени через единицу площади“ и дающей результат размерностью $\mathrm{W} / \mathrm{m}^{2}$. Автору данного Комментария не удалось понять, является ли это несоответствие размерностей опечаткой, сделанной несколько раз, или еще одной принципиальной ошибкой, влияющей на рассуждения авторов и представленные результаты. Причина состоит в том, что в примере, представленном в [1], мощность нагрева нити накаливания порядка $1 \mathrm{~W}$, но возможность согласования этой величины с вышеупомянутым значением $I$ не обсуждается. Естественно ожидать, что правильная размерность $I$ соответствует ограничению снизу на радиус шаровой молнии, тогда как неправильная требует, даже при оценках по порядку величины, обоснования существенного, на порядок, расхождения мощности нагрева с $I$, однако вопросы такого рода в [1] не затронуты.

При попытке обосновать возможность испускания шаровой молнией электромагнитного излучения, вызывающего свечение лампы накаливания, при сравнительно малых значениях $a$ в работе [1] сделана вторая однозначно идентифицируемая принципиальная ошибка. Выдвигается предположение, что заряд, ускоренное движение которого вызывает испускание обсуждаемого электромагнитного излучения, индуцируется в шаровой молнии внешним электрическим полем [1]. При этом делается следующее утверждение: „Величина заряда, индуцированного в электропроводной сфере радиуса $R$ внешним

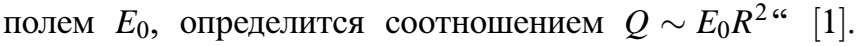
При вычислениях в системе СИ, используемой в работе [1], это утверждение верно только в случае, когда знак „ “ понимается в смысле пропорциональности, что видно из размерности. В обсуждаемой ситуации авторы [1] используют этот знак в смысле приближенного равенства или указания на порядок величины и приводят 
пример с $E_{0} \sim 1000 \mathrm{~V} / \mathrm{m}, R=0,15 \mathrm{~m}$ (эти величины служат как примеры напряженности приземного электрического поля в грозовую погоду и радиуса шаровой молнии соответственно) и $Q \sim 20$ С (таким образом, используется тот факт, что $\left.1000 \cdot 0.15^{2}=22.5 \approx 20\right)$. В книге [4] (задача 1 к параграфу 3 главы I, с. 31) рассмотрена поляризация проводящего незаряженного шара во внешнем однородном электрическом поле. Переписав полученное при этом выражение для поверхностной плотности электрического заряда в системе СИ и используя обозначения работы [1], легко показать, что на одной половине шара (или проводящей сферы) индуцируется положительный заряд $3 \pi \varepsilon_{0} E_{0} R^{2}$, который можно считать равным $Q$, а на другой - отрицательный заряд такой же абсолютной величины. При $E_{0} \approx 1000 \mathrm{~V} / \mathrm{m}$ и $R=0.15 \mathrm{~m}$ $3 \pi \varepsilon_{0} E_{0} R^{2} \approx 1.9 \cdot 10^{-9} \mathrm{C}$, что на десять порядков меньше соответствующей величины из [1].

Таким образом, авторы работы [1] не представили корректных оценок, способных служить для иллюстрации принципиальной возможности нагрева нити накаливания до визуально обнаружимого свечения токами, индуцированными электромагнитной волной, испускаемой шаровой молнией.

В заключение отметим, что установление одного или нескольких физических механизмов бесконтактного воздействия шаровой молнии на различные предметы и людей (см., например, обзор [5] и библиографию в $[1,5])$ необходимо для определения опасности шаровой молнии для людей и самолетов, а также для оптимизации лечения людей, пострадавших от воздействия шаровой молнии. Способность модели шаровой молнии объяснить надежные сообщения о таком воздействии может рассматриваться как один из критериев целесообразности ее экспериментальной проверки.

\section{Конфликт интересов}

Автор заявляет, что у него нет конфликта интересов.

\section{Список литературы}

[1] Григорьев А.И., Ширяева С.О. // ЖТФ. 2019. Т. 89. Вып. 1. C. 22-26. DOI: 10.21883/JTF.2019.01.46956.84-18 [Tech. Phys. 2019. Vol. 64. N 1. P. $14-18$. DOI: $10.1134 / \mathrm{S} 1063784219010146]$

[2] Фейнман Р., Лейтон Р., Сэндс М. Фейнмановские лекции по физике. Вып. 3,4, 3-е изд., М.: Мир, 1976. 496 с. [Feynman R.P., Leighton R.B., Sands M. The Feynman lectures on physics. Vol. 1. Reading, Massachusetts, Palo Alto, London: Addison-Wesley Publishing Company, Inc., 1963].

[3] Ахиезер А.И., Ахиезер И.А. Электромагнетизм и электромагнитные волны. М.: Высш. шк., 1985. 504 с.

[4] Ландау Л.Д., Лифииц Е.М. Электродинамика сплошных сред [Теоретическая физика. Том VIII], 2-е изд., перераб. и доп. Лифшицем Е.М. и Питаевским Л.П. М.: Наука, 1982. $624 \mathrm{c}$.

[5] Shmatov M.L., Stephan K.D. // J. Atmos. Sol. Ter. Phys. 2019. Vol. 195. P. 105115. DOI: 10.1016/j.jastp.2019.105115

\section{Мнение о работе Шматова М.Л. «Комментарий к статье А.И. Григорьева и С.О. Ширяевой „Оценка интенсивности электромагнитного излучения шаровой молнии“»}

К сожалению, оба замечания Шматова М.Л. справедливы, за них авторы весьма признательны.

Первое замечание, однако, мало что меняет в численных оценках, которые проводились по порядку величины, а само замечание (несмотря на грубую ошибку авторов) при его учете приводит к численному коэффициенту 3.

Второе замечание действительно существенно (перепутаны системы физических единиц): в итоге роль заряда, индуцированного внешним электрическим полем, в генерации электромагнитного излучения ШМ оказывается незначительной по сравнению с ролью собственного не скомпенсированного заряда.

И все-таки, авторы, признавая собственный прискорбный промах, полагают, что изложенный в работе материал представляет научный интерес, а скрупулезное отношение внимательного читателя позволило улучшить качество изучения рассмотренного в работе вопроса.

Григорьев А.И. 